\title{
Impressions d'Extrême-Orient
}

\section{Voler jusqu'à mon village natal des Noyers}

Une nouvelle de Su Tong 苏童

\section{Wen He}

\section{(2) OpenEdition}

\section{Journals}

Édition électronique

URL : http://journals.openedition.org/ideo/616

ISSN : 2107-027X

\section{Éditeur}

Université Aix-Marseille (AMU)

Ce document vous est offert par Aix-Marseille Université (AMU)

\section{Aix Marseille \\ universite \\ initlative dexullente}

Référence électronique

Wen He, « Voler jusqu'à mon village natal des Noyers », Impressions d'Extrême-Orient [En ligne],

7 | 2017, mis en ligne le 31 décembre 2017, consulté le 30 mars 2018. URL : http://

journals.openedition.org/ideo/616

Ce document a été généré automatiquement le 30 mars 2018.

\section{c) (i) $९$}

Les contenus de la revue Impressions d'Extrême-Orient sont mis à disposition selon les termes de la Licence Creative Commons Attribution - Pas d'Utilisation Commerciale - Pas de Modification 4.0 International. 


\title{
Voler jusqu'à mon village natal des Noyers
}

\author{
Une nouvelle de Su Tong 苏童
}

Wen He

\section{Présentation}

1 «Voler jusqu'à mon village natal des Noyers » («Feiyue wo de fengyangshu guxiang » 越我的枫杨树故乡) est une nouvelle écrite à la première personne par Su Tong 苏童 en 19871. Ce récit exprime une forte nostalgie pour un pays natal où le narrateur ne s'est jamais rendu. Celui-ci y évoque la mystérieuse disparition d'un petit oncle inconnu, mort l'année de sa naissance. C'est grâce aux souvenirs racontés par son grand-père que le narrateur a pu rêver et imaginer tout ce qui constitue son village natal imaginaire et accomplir sa vocation, en tant que descendant du clan Tong, de retrouver l'esprit de son petit oncle et le ramener en ville afin de rejoindre la famille dans ses rêves.

2 À travers l'écriture de Su Tong, on peut voir un mélange harmonieux de vie et de mort, de réalité et de rêve, d'envie et d'effroi, mais aussi des images représentatives chez Su Tong telles que les fleurs de pavot et la rivière. Dans ce monde rêvé, le rouge est la couleur la plus présente. C'est non seulement celle de la fleur de pavot, du feu, mais aussi celle du sang et de l'envie.

\section{Voler jusqu'à mon village natal des Noyers}

3 Jusqu'au début des années 1950, la région de mon pays natal des Noyers était encore recouverte de champs de fleurs de pavot, qui étaient rares dans le sud de la Chine. Au printemps, les plaines qui bordaient les deux côtés de la rivière étaient envahies en toute liberté par le cramoisi des fleurs, couche par couche, qui rayonnait d'une splendeur extraordinaire, comme d'énormes vagues rouges qui ballottaient ce village isolé, un souffle qui sentait le sang des villageois à l'instant de leur naissance et de leur mort. Mon petit oncle était encore à la campagne, selon les on-dit, il apparaissait et disparaissait 
avec une rapidité déconcertante comme un chien sauvage dans les meules de foin, dans les champs de pavot, les tas de fumier sec et parmi les grosses femmes et ne pensait pas à rentrer à la maison. Éloigné de mille lis, je pense souvent à lui, lui qui était assis au milieu des grandes fleurs rouges du pays natal : un gaillard de la campagne petit, fort et bronzé, qui faisait face aux villes du sud-ouest, un air étrange sur sa petite joue comme s'il voulait dormir, rire et aussi maudire, et qui chantait une profusion de chants populaires tout mélangés dont l'un était pour appeler son chien bien aimé :

« Toutou, toutou, faufile-toi jusqu'à moi,

Mène-moi aux maisons-grottes que j'embrasse de jolis minois. »

Mon grand-père habitait en ville. Il était courbé sous le poids de l'âge mais sa mémoire était toute fraîche. Au crépuscule, ses soupirs profonds voltigeaient comme de la poussière dans la maison. Il tardait à se coucher : «Je serai peut-être aveugle dès que je me réveillerai demain ». Donc il restait assis les yeux grands ouverts dans sa chambre que la nuit dévorait peu à peu. Paisible, rigoureux, il ressemblait à une ancienne statue d'aigle en bronze.

Dans la pupille élargie du grand-père plongé dans ses souvenirs, je pouvais voir mon petit oncle. Mon grand-père mélangeait son fiston avec une meute de chiens sauvages. Mon petit oncle du temps jadis était un vrai espiègle. Il adorait porter un chapeau à large bord comme les gens de la ville et errer, tout excentrique, dans les champs de fleurs de pavot. Un été, il jeta son chapeau dans la rivière et fut fasciné par une meute de chiens sauvages. Les villageois voyaient alors le petit maître du seigneur rester avec les chiens toute la journée, farfelu, ne ressemblant ni à un homme ni à un chien, causant un scandale insolite dans le village.

Mon grand-père injuria son plus jeune fils en ces termes : «Cette bête est ignorante des affaires humaines et ne comprend que la nature des chiens. » "Abandonnons-le à son sort, qu'il devienne aussi un chien!» disait-il, inévitablement triste en pensant à son espiègle rejeton. Je ne sais pendant combien de nuit profondes, plein de vitalité, mon petit oncle a suivi en chancelant les chiens qui couraient çà et là sur les diguettes des champs. Ses traces de pas qui se collaient aux empreintes de pattes en forme de galets des chiens se trouvaient dans tous les coins du village. Quelquefois, mon petit oncle se précipitait tout essoufflé chez quelqu'un pour demander de l'eau tandis que les chiens aboyaient sans cesse sur les terrains vagues aux alentours. Les villageois qui habitaient au bord de la rivière connaissaient tous mon petit oncle. En parlant de lui, ils croyaient tous qu'il était la réincarnation d'un esprit surnaturel et ignoraient s'il apporterait au village le bonheur ou le malheur.

Lors de la fête des défunts, quand les membres du clan, en rang d'oignons, se rendaient en foule au temple des ancêtres pour offrir des sacrifices, personne ne voyait l'ombre du petit oncle. Mon grand-père, fou de rage, tapa contre une assiette de fruits d'offrandes en se prosternant front contre terre devant les tablettes ancestrales. Il demanda d'une voix enrouée : «Mes ancêtres, manifestez-vous, est-ce le chien qui a séduit mon fils ou bien est-ce lui qui a séduit le chien?»

Mon grand-père avait prévu à son grand désespoir que l'âme excentrique et exécrable de l'oncle errerait dehors éternellement. Quelques décennies plus tard, chez nous en ville, mon grand-père, assis sur son petit lit en bambou spécialement fabriqué au village natal et devenu lisse et luisant, rebattait les oreilles de la famille avec l'abandon de petit oncle à l'arrivée de l'inondation. Lorsque le grand bateau en bois blanc rempli de quarante personnes de la famille et de leur fortune était sur le point de lever l'ancre, petit oncle et 
son chien arrivèrent au bord de l'eau l'un après l'autre. L'oncle demanda : «Où partezvous? » Personne ne lui répondit mais beaucoup de mains essayèrent de le tirer dans le bateau pendant un long moment. Ils n'y parvinrent pas et s'aperçurent que la corde attachée au mollet de ce garnement était étroitement liée à un grand chien sauvage. Quand mon grand-père sauta du bateau et voulut dénouer la corde, petit oncle cria à plein gosier et lui griffa le visage. En voyant que le grand-père était allé chercher une grande hache en l'insultant, petit oncle, fou de terreur, cria au chien : «Léopard, Léopard, fuis, fuis vite! » Le chien s'enfuit en effet à toutes jambes et la corde tira fort petit oncle, ils avaient l'air de deux petites bêtes s'échappant à toute vitesse devant le fusil du chasseur. Mon grand-père poussa une longue plainte face au ciel car il savait que le bateau devait partir et qu'il devait abandonner son chenapan.

"Je vois très bien le village natal, tant que mes yeux ne sont pas atteints de cécité, je vois tous les jours le village des Noyers. » dit grand-père. Le fond de son cœur triste était assez vaste pour que les fleurs de pavot rouges poussent en vagues et que petit oncle et son chien les foulent aux pieds.

Mon petit oncle mourut à la fin de la floraison des fleurs de pavot en 1956. Sa mort fut liée à un chien, à une femme et à d'autres choses bizarres. Depuis la mort du petit oncle, les fleurs de pavot ont totalement disparu au village et du riz étincelant et transparent comme des perles ainsi que du blé brillant comme de l'or poussent de la terre noire de làbas.

Je ne sais combien de fois j'ai rêvé de m'envoler vers mon lointain pays natal. Je me suis vu approcher de jour en jour d'une rivière trouble jaunâtre qui se dirigeait d'est en ouest, traverser la rivière pour arriver sur la rive gauche où se dressait une tornade dans les champs de fleurs de pavot, comme une vague rouge qui m'emportait vers mon village natal estompé.

7 Un jour, des drapeaux blancs funéraires flottèrent sur le village, un nuage de brume grisâtre s'éleva sur le toit de notre maison. Entourées de la brume, beaucoup de silhouettes couraient çà et là en sanglotant. Les gens étaient en plein désarroi, comme si la scène de l'inondation du village était réapparue. Ai-je vu ce désastre de mes propres yeux à travers des milliers de montagnes et de sources?

8 Ce jour-là était l'anniversaire de la mort de petit oncle. L'esprit de petit oncle, n'ayant pas trouvé sa place, continuait d'errer dans tout le village et semait le désordre dans ce bourg d'ordinaire si calme. Lorsque les villageois des Noyers, sous un vent légèrement parfumé par les fleurs de pavot, allèrent à la vieille maison des Tong pour assister aux funérailles, ils entendirent clairement un son semblable à celui d'une cloche funèbre. Il leur sembla avoir vu petit oncle s'asseoir, les jambes croisées, sur la meule à grains devant la porte de la vieille maison. La plante de ses grands pieds étaient couvertes de boue, de bouts d'herbe, et de crottes de chien. Les cinq orteils étaient bien écartés, tirés audacieusement vers le ciel. Souriant avec bonté et douceur, ses deux bras marqués de nombreuses petites bosses tiraient férocement la corde d'une cloche attachée à un vieil orme.

Le défunt petit oncle frappait sa propre cloche funèbre dont le son venu du Ciel ou du fond de la Terre faisait trembler de peur les villageois qui l'aimaient mais aussi le craignaient. Pendant l'anniversaire de la mort de petit oncle, beaucoup de personnes âgées et de femmes souffraient d'une affliction extrême. Elles criaient doucement au Soleil, à la Lune, aux étoiles, aux montagnes, aux eaux, aux herbes ainsi qu'aux arbres : «Emmenez-le, emmenez-le.» 
9 Auparavant, dans mon village natal, chacun après sa naissance possédait sa tablette d'esprit en bois de Phoebe qui se plaçait en hauteur dans la maison du chef du clan. Après la mort de son maitre, la tablette était incinérée et se transformait en oiseau de bonheur qui, d'une légèreté gracieuse, portait sur le dos le mort vers le ciel. Après avoir écouté mon grand-père raconter l'histoire de la tablette d'esprit, je sus que petit oncle était un malchanceux qui avait perdu sa tablette dont personne ne sut exactement le secret. Le bruit courait que, du fait de la nature libertine de petit oncle et de son viol des règles du village, le chef du clan, après avoir fait un rêve bizarre, avait couru à la rivière avec une tablette d'esprit dans les bras, tablette qu'il attacha avec une pierre afin de la faire sombrer jusqu'au fond de l'eau. Il est également dit que la folle Suizi des Noyers se faufila un jour dans la maison du chef du clan et vola la tablette d'esprit de petit oncle. Elle pénétra toute seule dans les champs sauvages, et alluma un feu où elle jeta l'esprit de petit oncle, toute folle, tantôt pleurant, tantôt riant. De ces rumeurs, mon grand-père ne croyait pas un seul mot. Ses yeux tristes fixés au plafond, il me dit: «Ton petit oncle a pris lui-même sa tablette d'esprit. Il l'a vendue à quelqu'un qui craignait la mort. Avec l'argent, il est allé boire et s'amuser avec les femmes, cela est sûr. À l'âge de quinze ou seize ans, il savait déjà commettre beaucoup de méfaits. "

Si ce n'était pas le cas, si la tablette d'esprit de petit oncle était encore bien dressée dans la maison du chef du clan, je me serais envolé jusqu'au lointain pays natal pour ramener l'esprit de petit oncle parmi la famille, en ville, où il n'est jamais venu.

Moi qui suis un descendant des Noyers vais entrer dans le temple ancestral du clan Tong et voir le chef du clan de quatre-vingt onze ans.

La maison du chef du clan est érigée sur un monticule de terre face au soleil, les fenêtres fermées. Seule l'entrée toute noire m'aspirera dans la maison. Sous une masse d'air plongée dans une obscurité qui sent le moisi et le pourri, j'aurai la tête qui tourne et les yeux éblouis. Inconsciemment, je tâtonnerai au hasard le long du mur pour chercher la corde de la lampe. Tout à coup, j'attraperai un fagot de planchettes en bambou couvert de poussière, horriblement lourd. Je le jetterai et continuerai de me cogner dans la pièce. Je finirai par me cogner sur le visage du chef du clan, ce qui me fera très mal, comme si j'avais heurté un vieil arbre de cent ans. Immédiatement, un filet de flamme se hissera devant mes yeux. Le chef de mon clan de quatre-vingt onze ans lèvera une bougie car il n'y a pas de lumière électrique dans sa maison. À l'aide de la lumière de la bougie, je parviendrai à voir clairement le visage saint et détaché du vieux chef du clan ainsi que son corps tout nu, ratatiné, vieux et aussi vigoureux. De ses yeux jaillira la flamme bleue d'une jeunesse encore plus vivace que la mienne. «Que cherches-tu?

- Dis-moi où est la tablette d'esprit de petit oncle.

- Elle a été perdue je ne sais quand. Une fois perdue, on ne peut plus la retrouver.»

Le chef du clan me sourira d'un air bienveillant à travers la lumière de la bougie alors que moi, n'ayant pas confiance en lui, je fouillerai parmi les tas de planchettes en bambou pour trouver le nom de petit oncle. Je sentirai que l'odeur des fleurs de pavot est de plus en plus intense, je verrai que les murs et le sol sont encombrés de grappes de fleurs de pavot séchées. Même le vieux chef du clan se métamorphosera en une énorme fleur de pavot, suffoquant le village calme et parfumé. Je chercherai tout en sueur. Je verrai les noms de tous les gens du village, parmi lesquels il y aura le nom de mon grand-père, de mon père et le mien, mais pas la tablette d'esprit de petit oncle.

«Qui l'a volée? ? Lorsque je demanderai à haute voix au vieux chef du clan, je verrai son visage s'enfoncer peu à peu dans l'obscurité. Il poussera un léger soupir, éteindra en 
soufflant la bougie dans sa main et me renverra hors de la maison. Tout confus, je descendrai du monticule pour rechercher les dernières traces de petit oncle dans le village. Je parviendrai, en m'appuyant sur ma sensibilité envers les chaussures noires en caoutchouc qu'avait portées petit oncle, à sentir son odeur, un mélange fétide de sueur et d'alcool.

Les chaussures noires en caoutchouc avaient été fabriquées dans des usines de notre ville. Le jour de l'anniversaire de ses soixante ans, mon grand-père, en voyant qu'il pleuvait à torrents derrière la fenêtre, pensa subitement à quelque chose et, sous la pluie, alla dans la rue acheter cette paire de chaussures noires en caoutchouc qui, enveloppées de trois couches de toile huilée, furent envoyées à petit oncle au pays natal, qui se trouvait à mille lis d'ici. Ce fut le seul cadeau que lui avait offert mon grand-père pendant toute sa vie.

11 J'entendis dire que c'était à la Fête des fantômes, soit le 15ème jour du septième mois lunaire, que petit oncle avait porté pour la première fois ces chaussures noires en caoutchouc. On ne savait pas quand la Fête des fantômes était devenue une fête où l'on brûlait des fleurs dans la région de Noyers. Chaque fois que ceux des générations précédentes qui y avaient habité se remémoraient cette fête, je me croyais au paradis. Ils disaient que portant ses chaussures en caoutchouc d'un noir de jais, petit oncle se mettait à côté d'un char tiré par un bœuf rempli de pavot, prêt au départ. Le bœuf, enduit partout d'huile d'arachide très parfumée et de pollen de fleurs de pavot, était attelé au char, merveilleux et éblouissant. C'était le moment le plus glorieux de petit oncle dans le village, selon eux. Il brandissait son long fouet en bambou, montait sur le char en levant une jambe, tapotait tendrement le ventre du bœuf avec ses grandes chaussures noires en caoutchouc. Tous les esprits dans le char, qu'ils soient grands ou petits, se mettaient en route avec petit oncle. Sous le ciel serein et bleu, la mèche commençait à brûler. Un voile de fumée rouge sombre s'élevait du char à bœufs qui galopait en rase campagne comme un nuage filant. Derrière petit oncle, les grands esprits et les petits se transformaient en tige, en bouton, en pétale dans la flamme. Ils se mettaient tous à s'exalter et à s'agiter, poussant aux quatre coins de la rase campagne des cris aigus et extraordinaires. On entendait rire de bonheur petit oncle. Avant que la flamme qui raccompagnait les esprits lèche son dos, petit oncle chantait, criait, joyeux comme un dieu.

Chaque année, c'était petit oncle qui servait de raccompagnateur d'esprits, ce qui semblait être la seule chose qu'il avait envie de faire au village. J'entendis aussi dire que les bœufs poussaient des cris tristes dès qu'ils voyaient les chaussures noires en caoutchouc car "l'humain parait très grand dans les yeux du bœuf ", les chaussures de petit oncle semblables à deux montagnes sinistres opprimaient les nerfs des bœufs. Il disait souvent aux gens qu'il avait entendu la malédiction collective lancée par les bœufs en passant par leur enclos. "Il ne mourra pas d'une bonne façon », avaient dit tous les bœufs des Noyers.

Les vieux bœufs qui avaient raccompagné les esprits apparurent à plusieurs reprises dans mes rêves où je vis nombre d'entre eux mourir sous les fesses de petit oncle. Les bêtes, étouffées par la flamme toxique des fleurs, perdaient connaissance. Bouleversés par l'ambiance de la Fête des fantômes, ils devenaient fous. Un taureau, après s'être échappé de l'entrave tirée par petit oncle, des fleurs et des fantômes, finissait par traverser la rivière du village. Je m'évertuais à me figurer le taureau tout en liberté et en gaieté, espérant qu'il pourrait se libérer de toutes les souffrances. Je voulais qu'il porte aussi une paire de chaussures noires en caoutchouc géantes.

Mon grand-père avait prédit que petit oncle terminerait sous les sabots d'un bœuf. Il se 
doutait que la paire de chaussures qu'il avait offertes à petit oncle deviendrait un portemalheur qui provoquerait beaucoup de jalousie et de haine. En 1956, la nouvelle de la mort de petit oncle à la campagne nous parvint. Il mourut dans la rivière, tout nu, portant seulement les chaussures noires en caoutchouc.

Né en 1956, j'étais un bébé beau et calme. Mais je vis clairement de mes propres yeux la veillée funèbre qui vivait dans mes souvenirs.

Le chant des cigales d'automne flottait dans les champs éclairés par la Lune. Le moulin en pierre de la vieille maison était entouré par une foule dense et noire de veilleurs silencieux, debout, figés comme des montagnes. De nombreux vieillards, femmes, enfants et hommes qui se tenaient les uns à côté des autres en désordre encerclaient et protégeaient le milieu d'un lotus - mon petit oncle mort. J'entendis un garçon blanc comme la neige frapper un tube de bambou. Lorsqu'un brin d'encens finissait de brûler, il tapotait trente-six fois le tube de bambou dont le bruit était accompagné par l'avancée de la nuit.

Plongé dans un chagrin simple issu de l'amour familial, je dormais dans mon berceau, sur le point de pleurer. Pour la première fois, je vis petit oncle qui était mort noyé, qui, tout bleu, les yeux grand ouverts, s'allongeait à côté de l'énorme meule en pierre du pays natal. La salle funèbre dans le lointain était à mille lis de moi, mais il me semblait qu'elle était installée à côté de mon berceau. Le petit moi, après avoir traversé les montagnes, les eaux, les villageois, les bêtes, rougis et ne cessai de souffler. La rivière où petit oncle s'était noyé était bien exposée sous mes yeux dont l'eau coulait en faisant « ouin ouin » sous la Lune. Les fleurs de pavot sans bornes sur la rive gauche ondulaient et vacillaient au vent, d'où jaillissait une envie infinie écarlate. Une sorte de tristesse solennelle à l'égard de la vie et de la mort se répandit dans tous les coins du monde. Touché par quelque chose de profond et de complexe, je me levai en vacillant du berceau, éclatai en sanglots face à la Lune derrière la fenêtre. Mon grand-père, mes parents et mes frères accoururent en panique. Ils virent que je pleurais à en perdre la raison et qu'un filet de lumière pure brillait de plus en plus dans mes yeux.

Vis-je aussi s'élever de l'eau l'esprit de petit oncle, tout fluorescent et qui flottait de la rive gauche vers la rive droite? Prévis-je que petit oncle ne serait pas en mesure d'aller au-delà de la rivière torrentueuse et trouble, ce qui formait une unification harmonieuse d'un mort et de ce monde qui me terrorisait?

Depuis de nombreuses années, j'ai toujours voulu trouver les témoins oculaires de la noyade de petit oncle, la femme folle Suizi et le chien sauvage. Mon grand-père se souvenait que petit oncle était un très bon nageur qui ne pouvait point se noyer même avec un poids en fer attaché autour du cou. Alors comment la folle Suizi parvint-elle à tuer petit oncle, lui qui nageait comme une anguille? Parmi les villageois des Noyers, personne ne se doutait de la noyade de petit oncle dans la rivière. Ils ne le surent qu'après avoir vu grimper vers la rive la folle Suizi, toute mouillée, tenant une chaussure en caoutchouc noir de jais dans la main. Tout le monde faisait sécher au soleil les graines de pavot sur la place devant leur maison, ne prenant pas garde à la rivière. Seul le chien sauvage de petit oncle avait tout vu. Il avait vu, tout en silence, les incessantes éclaboussures d'eau dans la rivière et l'ombre d'un homme et d'une femme qui étaient comme deux poissons lisses et nus. Personne n'avait entendu les aboiements du chien. Ils disaient que, si j'avais volé au-dessus du pays natal à cet instant-là, je n'aurais rien vu d'autre qu'un midi silencieux et paisible. Cependant, la mort de petit oncle me semblait un grand complot parfaitement ourdi, qui était toujours gravé dans mon cœur. 
Pendant les trois jours et trois nuits durant lesquels les villageois veillaient petit oncle, la folle Suizi, vêtue de sa tenue de deuil blanche, fit son apparition près de la meule en pierre où il y avait la salle funéraire, toute échevelée, hébétée, belle mais excentrique. Elle se mit à genoux à côté de la dépouille sans vie de petit oncle en contemplant avec plein de tendresse son visage aussi brillant que le saphir. Suizi était à moitié cachée par les billets funéraires qui s'entassaient sur le sol. Suite à un coup de vent nocturne qui souleva subitement les billets funéraires, les veilleurs aperçurent que son pied gauche était nu et que son pied droit portait cependant la chaussure noire en caoutchouc de petit oncle.

L'autre chaussure avait disparu. J'ignorais à partir de quel moment, ses chaussures s'étaient échappées de ses pieds crottés et avaient chacune pris leur route.

J'ai entendu parler de quelques histoires sur la folle Suizi. Dans la région des Noyers, au printemps, pas mal d'hommes la saisissaient par force dans les champs de fleurs de pavot où ils forniquaient en pleine nuit. Les hommes rentraient chez eux en courant rapidement après avoir tapoté les seins charnus de Suizi, la laissant dormir toute seule à poings fermés parmi les vagues de fleurs de pavot. Les gens qui travaillaient de bon matin dans les champs la trouvaient souvent là, dormant toute nue. Face au soleil levant, les lèvres légèrement ouvertes, d'innombrables gouttes de rosée scintillantes et fraîches pénétraient jusqu'au fond d'elle. Si on la regardait de loin, Suizi allongée le matin dans les champs de pavot ressemblait à un navire sans gouvernail flottant dans les vagues écarlates de fleurs de la rive gauche. J'ai également entendu dire qu'elle tombait enceinte tous les deux ans. Personne ne savait les dates de ses accouchements. Chaque fois qu'elle perdait les eaux, elle allait vers la rive en rampant. Les nouveau-nés tombaient dans l'eau et flottaient vers l'aval. Ces nouveau-nés étaient tous très beaux mais criaient comme des vieux, solitaires et mornes. Dans les villages de l'aval de la rivière, bien des enfants qui étaient arrivés au fil de l'eau grandirent peu à peu, comme du millet sauvage qui tuyautait, plein de vie. Ces enfants noirâtres et crasseux, vivaces et agiles apparurent à plusieurs reprises dans mes rêves. J'ai la vague impression qu'ils présentent une ressemblance frappante avec petit oncle décédé et qu'ils sont peut-être le cristal de son essence et de son sang, semé librement dans la terre noire et qui poussa, fleurit et fructifia.

16 Je rencontrerai le chien sauvage de petit oncle au bord de la rivière. J'entendrai derrière moi ses pas. Je sentirai les émanations fétides de sa fourrure qui deviendront de plus en plus fortes. Je m'accroupirai et tournerai la tête pour fixer mon regard furieux sur lui. Géant et malicieux, le chien lèvera ses pattes avant comme si c'était ses mains alors que les pattes arrière soutiendront tout son poids pour faire des gestes d'humain. Je verrai que sur son dos seront tombées plein de pétales écarlates de fleurs de pavot et que même ses yeux fumés par le feu deviendront deux agates.

De son vivant, petit oncle ne faisait qu'un avec le chien sauvage qui, lorsque petit oncle dormait profondément, se mettait à aboyer à pleine voix, les pattes sur son ventre décharné. Le chien était pour moi une femme dévergondée qui obsédait petit oncle tout au long de la journée, qui le mettait à plat et le tirait vers la rivière mortelle. Je soulèverai une pierre et me tiendrai face au chien pendant un long moment. Lorsque je lèverai la pierre au-dessus de ma tête, il émettra un cri triste du fond de sa gorge, se faufilera dans les champs de fleurs de pavot et s'y évanouira.

17 «Petit tonton petit tonton tue le chien dépêche-toi

Tue le chien et rentre avec moi » 
18 En poursuivant le chien sauvage le long de la rivière, je me rappellerai clairement les poèmes que j'avais écrits à petit oncle. Ce jour-là, en toute hâte, je chercherai petit oncle en tout lieu comme une mouche sans tête au village. Je verrai l'esprit de petit oncle jeter des éclats blancs devant moi qui me guideront pour accomplir mon voyage irréalisable au pays natal.

Sur le chemin, je découvrirai des paysages merveilleux au bord de la rivière. La roue à aubes enduite d'huile d'abrasin sur laquelle mon grand-père avait pédalé dans sa jeunesse tournera en craquant. Un homme déchargea entre les cuisses d'une femme sur la roue à aubes transmise par les ancêtres. L'eau dans le canal sera aussi stagnante que de la glace. A l'extrémité des champs, un taureau noir galopera à corps perdu, tandis que derrière se réuniront en foule des guêpes dans le ciel. Les guêpes, en bourdonnant, poursuivront les cornes gâtées du taureau qui se dirigera vers la rivière. Quand j'irai jusqu'à la rive gauche, je verrai de mes propres yeux la folle Suizi vêtue de la tenue de deuil blanche. Elle s'approchera pas à pas vers la rivière, chaussée d'une seule botte noire en caoutchouc. Lorsque l'eau atteindra sa poitrine opulente, elle lèvera son beau visage et baissera soudainement la tête jusqu'à toucher ses clavicules de son menton, les cheveux pendant à la surface de l'eau. Elle saisira fermement sa touffe de cheveux et la rincera dans l'eau sans cesse. Beaucoup de bulles rouges naîtront à la surface de l'eau ondulée. Peu à peu la moitié de la rivière deviendra rouge.

Tout sera du déjà-vu, ressemblant à ce dont j'ai rêvé chez moi en ville, sauf que l'esprit de petit oncle en bottes noires en caoutchouc qui était bronzé, robuste, maigre, petit et errant ne laissera aucune trace. Est-il en train de sourire ou de pleurer? Mon petit oncle !

Le huitième jour du huitième mois lunaire en 1956, la veille de l'enterrement de petit oncle, tous les villageois avaient parlé du mort qui avait perdu sa tablette d'esprit sans laquelle il ne pourrait pas rentrer au cimetière du clan. Afin de la retrouver, ils avaient cherché dans toutes les maisons du village et les champs et fouillé dans les habits de toutes les femmes qui avaient été avec petit oncle, en vain. Le village était en grand désordre. Petit oncle, allongé sur le moulin en pierre, avait enduré le tumulte que luimême avait produit. À l'heure du rat, le tube de bambou s'échappa brusquement des mains du petit veilleur, qui se mit ensuite à pleurer et à s'écrier que petit oncle mort avait ouvert les yeux, ses yeux qui étaient aussi rouges que les boutons de fleurs de pavot au printemps, et dans lesquels il avait vu une femme et un chien.

Ils disaient tous que c'était la folle Suizi et le chien sauvage qui s'étaient faufilés dans les yeux de petit oncle. Mon grand-père disait la même chose. Lors de cette dernière nuit de la veillée, mon grand-père entendit les cris du garçon, malgré une longue distance de mille lis, tandis qu'il fabriquait avec le plus grand soin une planchette en bambou. Si bien taillée, avec le nom de petit oncle gravé dessus, elle était parfaitement identique aux tablettes d'esprit dans la maison du chef du clan. Après avoir fini cela, il rit à quelques reprises et ensuite s'étouffa de sanglots. Il grimpa à une échelle pour monter sur le toit de la maison d'où il contempla notre ville. Éperdu, il déraisonnait comme un sorcier. Le toit en trembla longtemps sous son supplice. Ce jour-là, les piétons qui passèrent en bas de la maison aperçurent tous un feu follet qui se jeta du toit jusqu'à la rue où il brûla en crépitant et cracha une flamme bleue haute d'un pied. Exhalant un parfum extrêmement frais, le feu follet chanta et dansa sans aucune retenue sur la route bétonnée. Cela dura tout le crépuscule : «Ramène petit oncle à la maison. »

20 Au printemps de l'année d'avant, mon grand-père, assis sur son petit lit en bambou venu des Noyers, connaissait peu à peu les dernières heures de son agonie. Mon petit oncle 
décédé depuis plusieurs années tourmentait le fil confus de ses pensées. Mon grand-père, ne pouvant plus endurer cela, appuya ma tête peinée contre sa poitrine et me dit d'une voix basse : « Ramène petit oncle. »

21 Je m'envolerai, en fin de compte, jusqu'à mon village natal afin d'accomplir le vœu inachevé des trois générations de ma famille. Mais jamais personne ne m'a expliqué, pourquoi les pavots rouges s'étendant sans borne avaient-ils été plantés le long de la rive gauche? Les pavots rouges qui représentaient la vie et la mort, pourquoi n'existent-ils plus? Lorsque je porterai sur le dos mon petit oncle qui avait abandonné ce monde il y a longtemps pour fuir le village, je reverrai les champs de pavots du temps jadis. Ce sera un soir chaud et lourd où la Lune tombera chaque seconde. Elle sera si brûlante et bouillonnante qu'elle pourra presque nous brûler et nous carboniser. Les courants nocturnes d'un rouge sombre du pays natal s'agiteront éternellement et, avec les vagues nocturnes de fleurs de pavot, entoureront les exilés sous la nuit profonde. Sur combien de grenouilles grises marcherai-je? Elles coasseront à haute voix et se mettront frénétiquement à nous poursuivre sur les diguettes des champs.

J'entendrai le brouhaha de la foule du village, qui s'éclairera subitement dans tous les coins. Une meute de chiens surviendra, les villageois me courront après pour arracher l'esprit de petit oncle qui naquit et mourut ici. Errant aux champs, le vieux chien laissé par petit oncle aura atteint son immortalité. Ses yeux aussi brillants que les étoiles filantes au ciel nocturne, il se jettera de toutes ses forces sur nous. Le bruit des hommes, des chiens, de la nature me suivant, la Lune bouillante tombant jusqu'à la poitrine bronzée du mort calme et paisible, le parent que je porterai sur le dos sera un volcan millénaire.

Pendant ma nuit de fuite, une folle, très loin, mettra un autre enfant au monde. Tout le monde entendra ses sanglots solitaires et mornes qui cacheront les vicissitudes de ce village millénaire. Parviendrai-je, à travers ces cris d'une nouvelle vie, à franchir le long champ de la rive gauche ainsi que la rivière?

Sous les toits de cette ville vivent beaucoup de familles immigrées de la campagne. Chaque nuit, leurs ronflements s'entrecroisent. Ils ont chacun leur souci et leur rêve. Si vous êtes comme moi qui fais des rêves étranges depuis l'enfance, vous rêverez de votre pays natal, de votre famille et de vos parents. Comme si vous étiez sur un radeau en bambou qui descend au fil de l'eau dans une rivière innée, et que vous jetiez un regard rétrospectif sur votre pays natal lointain.

\section{NOTES}

1. On a pu lire une nouvelle de cet auteur dans le numéro un d'Impressions d'Extrême-Orient. Voir à l'URL : http://ideo.revues.org/75 Nous y renvoyons le lecteur. La nouvelle dont nous fournissons une traduction inédite a été publiée dans le recueil Shennü feng 神女峰 (Mont de la déesse). Shanghai : Shanghai wenyi, 2015, p. 178-190 


\section{AUTEUR}

WEN HE

IrAsia 